\title{
Design and Coupled Electro-Fluidic Simulation of a Novel Dielectrophoretic Microfilter*
}

\author{
O.T. NEDELCU ${ }^{\dagger}$ \\ National Institute for Research and Development in Microtechnologies \\ 126A Erou Iancu Nicolae Str., 077190, Bucharest, Romania
}

(Received October 31, 2011)

\begin{abstract}
In this paper a design of a new dielectrophoretic microfilter is proposed, based on electrodes configuration having stairs geometry that multiply the opposite points of electrodes where minimal and maximal values of electric field are obtained. The results of electrostatic simulation show very well defined regions of extremes values where particles can be separated. A model for particle-fluid interaction is used in momentum equation as reaction body force depending on electric field and particle concentration. The fluid flow and particles separation were simulated for positive dielectrophoresis, based on electrostatic results by means of dielectrophoretic velocity and reaction force acting on suspending media. The distribution of particle concentration depends on dielectrophoretic velocity and flow velocity, but also it influences the flow velocity, hence these fields were solved in coupled approach. The resulting flow profile, streamlines and concentration distribution demonstrate that particle migration influences the surrounding fluid and leads to modified velocity distribution by comparison to classical fluid flow.
\end{abstract}

PACS: 47.54.Fj, 47.57.jd

\section{Introduction}

Dielectrophoretic (DEP) microfilters are based on the movement of polarisable particles in non-uniform fields and have applications as trapping and separation of cells, viruses or macromolecules [1-6]. The advanced microfabrication techniques have led to rapid development of various types of microelectrodes that can manipulate micro and nanoparticles. The dielectrophoretic force acting on particles depends of non-uniformity of electric field and it is a short range force since the electric field gradient decrease rapidly from an electrode edge [7], particularly in the volume of separation channel. The configuration of electrodes is therefore an essential issue in developing electric field gradients able to separate the microparticles efficiently.

Reported DEP microfilters use castellated [8], interdigitated [1], or polynomial microelectrode arrays [9, 10]. In such microfilters, the fluid flow with particles in the separation channels of DEP microfilters is described using the Navier-Stokes equation system, but the equations are applied to fluid media properties, without taking into account the presence of particles. Depending on concentration, suspensions can modify flow parameters through density of solution composed by carrier fluid and the particles and also through reaction force acting on fluid as response to DEP migration of particles.

\footnotetext{
* Paper presented at EMRS Fall Meeting, Symposium F - Biological Sensors, Warsaw, Poland, 2011

$\dagger$ e-mail: oana.nedelcu@imt.ro
}

\section{Theory and models}

\subsection{Dielectrophoretic force}

Dielectric particles placed in a non-uniform AC electric field experience a force that depends on the applied field intensity and frequency, properties of fluid media and particle (permittivity, conductivity), and particle dimension and form (7). For spherical particles, the time averaged DEP force can be written

$$
\boldsymbol{F}_{\mathrm{DEP}}=\frac{1}{2} V_{\mathrm{P}} \operatorname{Re}\left(\alpha^{*}\right) \nabla\left|\boldsymbol{E}_{\mathrm{RMS}}\right|^{2},
$$

where $\alpha^{*}$ is the particle polarisability, depending on complex permittivity of medium and particles, and angular frequency of electric field $\omega, V_{\mathrm{p}}$ is the volume of one particle, and $\boldsymbol{E}_{\mathrm{RMS}}$ is the root mean square of electric field. Other parameters used in evaluation of particles migration capability are DEP mobility $\mu_{\mathrm{DEP}}$ and DEP velocity $\boldsymbol{v}_{\text {DEP }}$ given by

$$
\begin{aligned}
& \mu_{\mathrm{DEP}}=V_{\mathrm{p}} \alpha / 4 \varphi, \\
& \boldsymbol{v}_{\mathrm{DEP}}=\mu_{\mathrm{DEP}} \nabla|\boldsymbol{E}|^{2},
\end{aligned}
$$

where $\varphi=6 \pi \eta R$ is the Stokes factor, $\alpha^{*}=\operatorname{Re}\left(\alpha^{*}\right), \eta$ is the fluid viscosity and $R$ is the particle radius. The frequency of electric field is the main parameter that induces the type of mobility: positive mobility separates the particles in regions with high electric field, while particles having negative mobility are separated in regions with high electric field. 


\subsection{Fluid-particle interaction}

The particles move under combined effects of solution flow and DEP force. The relative movement of particles related to fluid is DEP migration that influences the fluid flow through a reaction body force. Knowing the DEP force acting on a single particle, for $N$ particles in an elementary volume $V$, the total DEP force will be

$$
\boldsymbol{F}_{\text {DEP_Total }}=N \boldsymbol{F}_{\text {DEP }} \text {. }
$$

This force divided by $V$ leads to the body force that acts on particles in volume $V$, expressed by force density

$$
\boldsymbol{f}_{\mathrm{DEP}}(\boldsymbol{x})=c(\boldsymbol{x}) N_{\mathrm{A}} \boldsymbol{F}_{\mathrm{DEP}},
$$

where $c(\boldsymbol{x})$ is the molar concentration. The reaction force that acts on the fluid will be

$$
\boldsymbol{f}_{\mathrm{R}}(\boldsymbol{x})=-\boldsymbol{f}_{\mathrm{DEP}}(\boldsymbol{x}) .
$$

This force depending on concentration and particle volume will modify the fluid flow profile by comparison to classical fluid flow.

\section{Results and discussion}

The basic configuration of microfilter consists in a channel separation $(330 \mu \mathrm{m} \times 100 \mu \mathrm{m} \times 20 \mu \mathrm{m})$, and planar electrodes on the top surface and it is illustrated in Fig. 1. The "stairs" geometry of the electrodes is designed to multiply the opposite points of electrodes where minimal and maximal values of electric field are obtained. The minimal dimensions are $5 \mu \mathrm{m}$ gap and $10 \mu \mathrm{m}$ width.

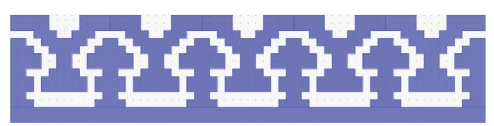

Fig. 1. Geometrical configuration of stairs electrodes: minimal width: $10 \mu \mathrm{m}$; minimal gap: $5 \mu \mathrm{m}$; channel: $330 \mu \mathrm{m} \times 100 \mu \mathrm{m} \times 20 \mu \mathrm{m}$.

Electrostatic simulation (Comsol Multiphysics) was performed for $10 \mathrm{~V}$ and $20 \mathrm{~V}$ applied voltage between electrodes. The intensity obtained in the domain varies between $89 \mathrm{~V} / \mathrm{m}-3.93 \times 10^{6} \mathrm{~V} / \mathrm{m}$ for $10 \mathrm{~V}$ and $178 \mathrm{~V} / \mathrm{m}-$ $7.86 \times 10^{6} \mathrm{~V} / \mathrm{m}$ for $20 \mathrm{~V}$. The results are presented in Figs. 2-4 and show very well defined regions of extreme values where particles can be separated. The maximal values are obtained in the regions of the opposite corners.

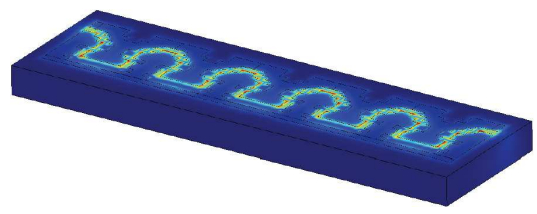

Fig. 2. Electric field distribution in domain (10 V applied).

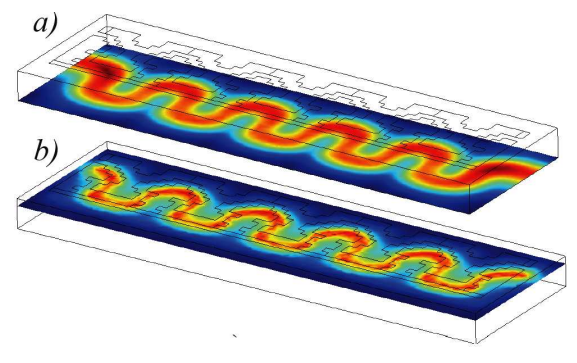

Fig. 3. Electric field distribution in $X-Y$ plane sections: (a) section opposite to electrodes surface; (b) section near electrodes surface.

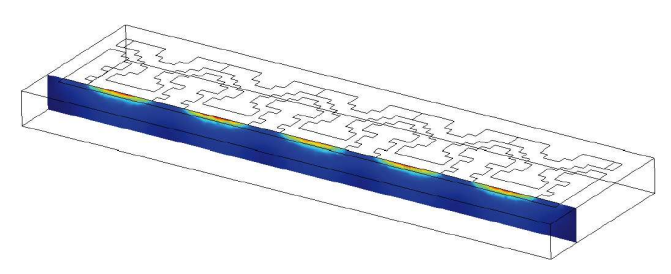

Fig. 4. Electric field distribution in $X-Z$ plane section.

Figure 3 illustrates the electric field distribution in two sections: opposite to electrodes surface and near electrodes surface. For these regions, the maximal values $\left(1.93 \times 10^{5} \mathrm{~V} / \mathrm{m}\right.$ and $6.98 \times 10^{5} \mathrm{~V} / \mathrm{m}$, respectively) are lower than global maximal value, but the gradient leads to a net DEP force oriented from edges to center. In Fig. 4 the distribution in vertical section is presented; the electric field gradient is higher and oriented to the electrodes surface.

In positive DEP, this type of electric field distribution will separate the particles from whole volume both in horizontal section from edges to center and in vertical sections to electrode surface.

Simulation of solution flow and particles separation was performed, considering deionized water as carrier fluid (relative permittivity 80 , conductivity $6.6 \times$ $10^{-5} \mathrm{~S} / \mathrm{m}$ ) and polystyrene microspheres (conductivity $2 \times 10^{-4} \mathrm{~S} / \mathrm{m}$, relative permittivity 2.5 ). The previous results obtained for electric field distribution were used to find the DEP mobility and velocity, and reaction force acting on fluid corresponding to $10 \mathrm{~V}$ and $100 \mathrm{~Hz}$ frequency that induces positive DEP. The reaction force was introduced in momentum equation as body force and the DEP velocity was set as relative velocity between particles and fluid in diffusion equation. Particles having $1 \mu \mathrm{m}$ in diameter were considered, with an inlet concentration of $1 \mathrm{nM}$, leading to a volume fraction of 0.31 . The analyses were performed for various boundary conditions in pressure drop between inlet and outlet: $\Delta P=10-100 \mathrm{~Pa}$.

In Fig. 5 the result in particles concentration is presented for case of flow at $100 \mathrm{~Pa}$ pressure drop. The maximum value (0.62 volume fraction) is reached in the regions of opposite corners of electrodes. For lower pressure drops the concentration is higher in the first part of 
the channel since the contribution of advection velocity is lower, while the DEP velocity is the same. The concentration values decrease below 0.1 in terms of volume fraction near the surface opposite to electrodes.

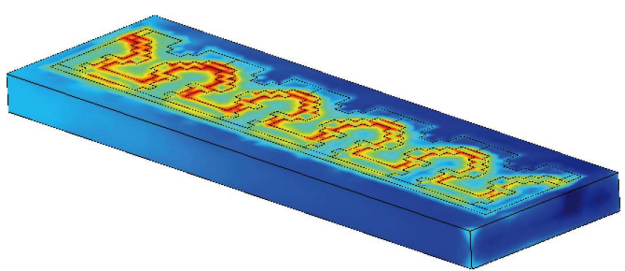

Fig. 5. Distribution of particles concentration for positive $\mathrm{DEP}$ and flow at $\Delta P=100 \mathrm{~Pa}$.

Figure 6 shows the velocity orientation of carrier fluid (uniform vectors representation). By comparison to classical flow with no body forces, where direction of flow is oriented along longitudinal channel axis, the reaction force changes the orientation to the opposite sense of particle migration.

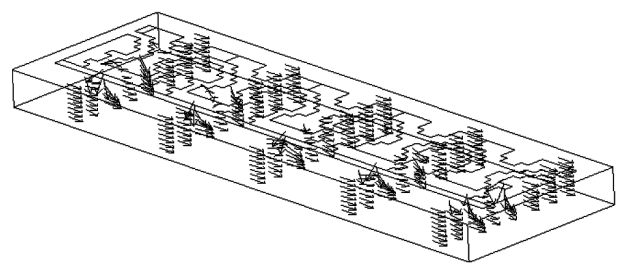

Fig. 6. Fluid velocity orientation at $\Delta P=100 \mathrm{~Pa}$.

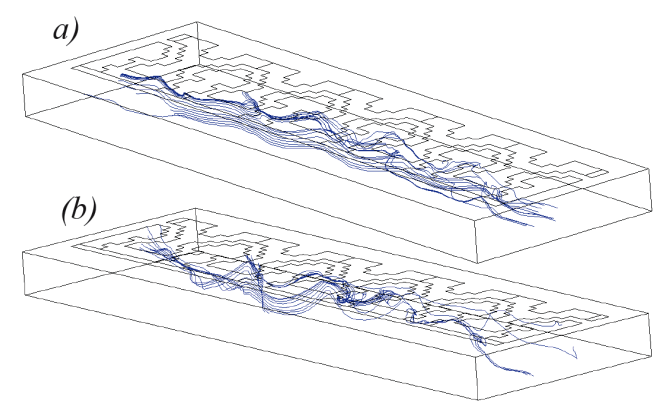

Fig. 7. Streamlines related to carrier fluid velocity: (a) $\Delta P=100 \mathrm{~Pa}$; (b) $\Delta P=50 \mathrm{~Pa}$.

In Fig. 7 streamlines of carrier fluid, passing through points at $100 \mu \mathrm{m}$ from inlet and middle width, illustrate the influence of DEP migration on fluid flow. At constant electrical parameters, particularly DEP force, this influence increases as the pressure drop decreases.

\section{Conclusions}

A new configuration of dielectrophoretic microfilter was proposed and simulated, based on stairs geometry of the electrodes. The induced gradient of electric field creates DEP force in both horizontal and vertical direction in fluid domain and increases the separation effect. A body force that models the fluid-particles interaction was used in flow analysis; this is a reaction force that acts on fluid as effect of dielectrophoretic migration of particles. Flow velocity and particles concentration are coupled parameters since concentration influence flow velocity by means of reaction force, and flow velocity has contribution on particles migration. The velocity and streamlines of carrier fluid show that fluid tends to change its flow direction in the opposite sense of particle migration; this effect depends on particle concentration, dielectrophoretic force and pressure drop along the channel.

\section{Acknowledgments}

The research presented in this paper is supported by the Sectorial Operational Programme Human Resources Development (SOP HRD), financed from the European Social Fund and by the Romanian Government under the contract number POSDRU/89/1.5/S/63700.

\section{References}

[1] Y. Ai, A. Beskok, D.T. Gauthier, S.W. Joo, S. Qian, Biomicrofluidics 3, 044110 (2009).

[2] Y. Kang, D. Li, Microfluid Nanofluid 6, 431 (2009).

[3] F.F. Becker, X.B. Wang, Y. Huang, R. Pethig, J. Vykoukal, P.R.C. Gascoyne, Proc. Natl. Acad. Sci. USA 92, 860 (1995).

[4] P.R.C. Gascoyne, Y. Huang, R. Pethig, J. Vykoukal, F.F. Becker, Meas. Sci. Technol. 3, 439 (1992).

[5] Y. Huang, R. Holzel, R. Pethig, X.B. Wang, Phys. Med. Biol. 37, 1499 (1992).

[6] R. Pethig, G.H. Markx, Trends Biotech. 15, 426 (1997).

[7] Encyclopedia of Microfluidics and Nanofluidics, Ed. D. Li, Vol. 2, Springer, New York 2008.

[8] N.G. Green, H. Morgan, J. Phys. D, Appl. Phys. 31, 25 (1998).

[9] N.G. Green, A. Ramos, H. Morgan, J. Phys. D, Appl. Phys. 33, 632 (2000).

[10] A. Castellanos, A. Ramos, A. Gonzalez, N.G. Green, H. Morgan, J. Phys. D, Appl. Phys. 36, 2584 (2003). 